\title{
Evidence of Daytime Variation of Asymmetric Dimethylarginine: An Intervention Study with Rosiglitazon in Type 2 Diabetes
}

\author{
Martin Carlsson, Torbjörn Lindström, Fredrik Nyström, Ingela Nilsson, Lars Brudin and Pär Wanby*
}

Department of Medicine, Kalmar County Hospital, SE-391 85 Kalmar, Sweden

\begin{abstract}
Background: Asymmetric dimethylarginine (ADMA) has in some, but not all studies been associated with insulin resistance (IR). We wanted to challenge the hypothesis that plasma ADMA levels are associated with IR in an intervention study using an insulin sensitizing drug. Another aim of the study was to study daytime ADMA variation and if food intake influence ADMA concentration.

Methods: Nine patients with diet treated type 2 diabetes were investigated with daytime profiles of ADMA ( 8 am-5 pm) before and during treatment with rosiglitazone for 8 weeks. A control group matched for age and sex underwent a similar investigation at baseline.

Results: After treatment with rosiglitazone, ADMA (0.63- $0.64 \mu \mathrm{mmol} / \mathrm{L} ; \mathrm{p}=0.26)$ and homocysteine $(10.3-10.6 \mu \mathrm{mol} / \mathrm{L}$; $\mathrm{p}=0.61)$ concentrations did not change. Postprandial $(10 \mathrm{am}-5 \mathrm{pm})$ ADMA concentrations were $10 \%$ higher than fasting morning levels $(\mathrm{p}=0.006)$ and this difference was similar for controls and diabetes patients both pre and post treatment with rosiglitazone.

Conclusions: Treatment with rosiglitazone aiming to improve insulin sensitivity did not affect ADMA concentration in type 2 diabetes patients.

The ADMA daytime variation and the relation to food intake appear to be a novel finding and should be considered in future studies.
\end{abstract}

Keywords: ADMA, insulin resistance, oxidized-LDL rosiglitazone, postprandial, circadian.

\section{INTRODUCTION}

Asymmetric dimethylarginine (ADMA) is a powerful endogenous competitive inhibitor of nitric oxide synthase (NOS) [1] and by reducing NO bioavailability ADMA has been implicated in the development of endothelial dysfunction, an integral part of the process leading to atherosclerosis [2]. Numerous studies have demonstrated elevated plasma concentrations of ADMA in clinical states associated with increased risk for cardiovascular disease. These conditions include insulin resistance, diabetes mellitus, hypertension, hypercholesterolemia, renal failure, and ageing [3-8]. Insulin resistance is characterized by reduced skeletal muscle [9] and hepatic glucose disposal, and can be provoked in healthy subjects by prolonged exposure to non-esterified fatty acids (NEFA) [10].

Thiazolidinediones (TZDs), agonists of peroxisome proliferator-activated receptor-gamma (PPARgamma), reduce NEFA plasma levels in patients with type 2 diabetes and improve insulin sensitivity. Rosiglitazone, a PPARgamma agonist, can enhance NEFA uptake into cells by increasing plasma triglyceride lipolysis and transendothelial NEFA transport [11].

*Address correspondence to this author at the Department of Medicine, Kalmar County Hospital, SE-391 85 Kalmar, Sweden; Tel: +46 48044 8204; Fax: +46 480 81998; E-mail: PerW@LTKalmar.se
Furthermore, plasma levels of ADMA fell significantly in a study by Stühlinger, in which seven nondiabetic but insulin resistant individuals were treated with rosiglitazone [12].

The plasma NEFA concentrations have a circadian rhythm related to insulin concentrations. Little seems to be known about a diurnal variation of ADMA.

The primary purpose of the study was to determine whether increased insulin sensitivity and a reduction of NEFA levels by rosiglitazone could influence ADMA concentrations in type 2 diabetes patients. The second objective was to study the daytime plasma profile of ADMA in diabetes patients and healthy controls and to interpret food intake as a factor contributing to possible ADMA variation.

\section{MATERIALS AND METHODOLOGY}

\section{Subjects}

Nine patients with Type 2 diabetes but without antihyperglycemic or lipid-lowering drugs were included in the study. The study subjects were mainly recruited via newspaper advertisements, and matched for age, sex and body mass index (BMI). Eight healthy non-smoking volunteers matched for age and sex were also recruited. The medical history of all subjects concerning, diabetes, hypertension and other cardiovascular diseases, and smoking was obtained by questionnaire. A history of hypertension was defined as the use of antihypertensive drugs or blood pressure more than 
$140 / 90 \mathrm{mmHg}$ at least in two separate determinations and diabetes mellitus as diet treatment only and/or a fasting plasma glucose value $\geq 7.0 \mathrm{mmol} / 1$.

The study was conducted at the County Hospital of Kalmar, Sweden. During the one or two days of in-hospital investigations subjects were given standardized meals with a calorie-content calculated from a modification of the equation of Schofield [13]. For the remaining study period the subjects were instructed not to change their normal diet or amount of physical activity. Fasting laboratory samples for all 17 subjects (lipids, glucose, HbAIc, liver function tests, homocystein, oxidized-LDL-cholesterol (oxLDL), NEFA and ADMA) were measured from venous blood samples after an overnight fast of 12 hours. The subjects were hereafter given breakfast and they were then investigated for 9 hours with blood samples (NEFAs, ADMA, glucose and lipids) at $8 \mathrm{am}, 10 \mathrm{am}, 11 \mathrm{am}$. Lunch was served at noon. Blood samples were then again taken at $3 \mathrm{pm}$ and $5 \mathrm{pm}$. In the second part, the subjects with diabetes were treated with rosiglitazone ( $4 \mathrm{mg}$ twice daily) for eight weeks and the same investigations as at baseline were then repeated in the patients with diabetes.

All participants gave informed consent. The study was approved by the Ethical Committee of Linköping University, Sweden, and was performed in accordance with the Declaration of Helsinki.

\section{Anthropometrics and Blood Pressure}

Weight and height were measured with the subjects in light clothing without shoes. Body mass index (BMI) was calculated as $\mathrm{kg} / \mathrm{m}^{2}$. Systolic and diastolic blood pressures were measured with the subject in the supine position. Abdominal supine sagittal diameter was measured with a calliper.

\section{Assays}

Methods: Samples for analysis of ADMA and oxLDL were collected in EDTA-tubes and centrifuged at $2000 \times \mathrm{g}$ for 10 minutes. Plasma was stored in $-70{ }^{\circ} \mathrm{C}$ until analysis. Blood samples for NEFA analysis were collected in tubes without additives. The blood was cooled in ice water for 30 min before centrifugation at $1500 \mathrm{~g}$ for $15 \mathrm{~min}$ at $+4{ }^{\circ} \mathrm{C}$. The serum was separated and stored at $-70{ }^{\circ} \mathrm{C}$ until analysis. HbA1c were analyzed on a Cobas Integra 700 (Roche). Plasma glucose, total-cholesterol, HDL-cholesterol, triglyceride and creatinine concentrations were analysed on a Vitros 5,1 FS / 350 (Ortho Clinical Diagnostics). Fasting plasma LDL-cholesterol concentration was calculated with the use of Friedewald's formula. NEFA were analyzed on a Cobas MIRA S (Roche) using NEFA-HR(2)-kit and controls (Wako Chemicals GmbH, Neuss, Germany). OxLDL and ADMA were assessed using ELISA-kits (Immundiagnostik AG, Bensheim, Germany for oxLDL and DLD Diagnostika $\mathrm{GmbH}$, Hamburg, Germany for ADMA).

Intra- and interassay variation and the methodological error: The intra-assay-coefficient of variation for oxLDL was $4.5 \%$ at our laboratory, the total-assay-coefficient of variation was $5.2 \%$ and the methodological error
(Dahlberg's equation using duplicate measurements) [14] was $6.3 \%$ (coefficient of variation). Corresponding values for ADMA were for the intra-assay-coefficient $13.4 \%$, for the total-assay-coefficient $15.3 \%$ and for the methodological error $7.3 \%$. The specified sensitivity for the ELISA methods was for ADMA $0.05 \mu \mathrm{mol} / \mathrm{L}$ and for oxLDL $4.13 \mathrm{ng} / \mathrm{mL}$.

\section{Statistical Analysis}

The results for continuous distributed variables are given as mean $\pm \mathrm{SD}$ and for categorical variables as percentages. Group differences and follow up differences were analysed using non-parametric tests (Mann-Whitney U-test for group differences and Wilcoxon's matched pairs test for differences between baseline and rosiglitazone treatment). Correlations were analysed using Spearman's rank correlation. Group differences and diurnal differences of ADMA concentration as well as differences pre post rosiglitazone were analysed with repeated measures ANOVA. The analyses were performed with STATISTICA (versions 8, StatSoft ${ }^{\circledR}$, Tulsa, USA) and p-values $\leq 0.05$ were considered statistically significant.

\section{RESULTS}

The patients with diabetes had higher measures for hip, waist and abdomen compared to the controls (Table 1). Patients with diabetes and controls, as expected, also differed in HbA1c and glucose at baseline (Table 2). No differences between the two groups were found in other parameters at baseline.

\section{Daytime Diurnal Variations in Patients with Diabetes and in Controls}

Six patients with diabetes and five controls were available for diurnal analyses. On average, postprandial ADMA concentrations (10 am - $5 \mathrm{pm})$ were $10 \%$ higher than fasting morning levels (Fig. 1); $\mathrm{p}=0.006$ ) and, although not statistically significant for the individual groups, the difference was similar for diabetes patients both pre $(11 \%)$, and post rosiglitazone (9\%) and controls (11\%), allowing the three groups to be combined in order to increase the statistical power (Fig. 2). Even when taking the diurnal variation into account, no associations were found between NEFA and ADMA levels $(\mathrm{n}=105, \mathrm{r}=-0.16, \mathrm{p}=0.10)$.

\section{Changes After Rosiglitazone Treatment (Patients with Diabetes)}

Data on metabolic parameters are summarised in Table 2.

Mean ADMA concentration after treatment $(0.59 \pm 0.07$ $\mu \mathrm{mol} / \mathrm{L}$ at 08 am and $0.64 \pm 0.07$ for the rest of the day) did not differ significantly from corresponding baseline values $(0.56 \pm 0.041$ for 08 am and $0.62 \pm 0.071$ for the rest of the day; $p=0.575)$ (Fig. 1).

\section{DISCUSSION}

Our data do not support a correlation between levels of ADMA and insulin-sensitivity, suggesting a more complex role for ADMA in diabetes than in atherosclerotic disease. At baseline no correlation between ADMA and NEFA was found. As expected, the intervention with rosiglitazone 
Table 1. Clinical Characteristics of Subjects with Diabetes and Controls at Baseline

\begin{tabular}{|c|c|c|c|}
\hline & Patients with Diabetes $(\mathrm{n}=9)$ & Controls $(n=8)$ & Difference \\
\hline Parameter & Median (Range) & Median (Range) & p-Value \\
\hline Age & $67(43-74)$ & $62(40-74)$ & 0.673 \\
\hline Height $(\mathrm{cm})$ & $170(156-187)$ & $172(164-186)$ & 0.721 \\
\hline Weight $(\mathrm{kg})$ & $86(68-104)$ & $77(54-106)$ & 0.442 \\
\hline $\operatorname{BMI}\left(\mathrm{kg} / \mathrm{m}^{2}\right)$ & $30(26-35)$ & $26(20-33)$ & 0.083 \\
\hline $\mathrm{SBP}(\mathrm{mmHg})$ & $140(120-150)$ & $130(110-150)$ & 0.209 \\
\hline $\mathrm{DBP}(\mathrm{mmHg})$ & $80(75-104)$ & $75(70-84)$ & 0.053 \\
\hline Waist (cm) & $103(95-115)$ & $88(72-116)$ & 0.038 \\
\hline Hip (cm) & $109(103-122)$ & $103(91-106)$ & 0.014 \\
\hline Sagittal abdominal diameter $(\mathrm{cm})$ & $25(22-28)$ & $22(15-30)$ & 0.050 \\
\hline
\end{tabular}

SBP and DBP =systolic and diastolic blood pressures. Waist and hip are waist and hip circumference.

Table 2. Data at Baseline in Patients with Diabetes and Controls and After Treatment with Rosiglitazone. Mean \pm SD

\begin{tabular}{|c|c|c|c|c|c|c|}
\hline \multirow[b]{2}{*}{ Parameter } & \multicolumn{3}{|c|}{ Diabetics (9) } & \multicolumn{2}{|r|}{ Controls (8) } & \multirow{2}{*}{$\begin{array}{c}\text { Controls vs Patients with } \\
\text { Diabetes (Baseline) } \\
\mathbf{p}^{* *}\end{array}$} \\
\hline & Baseline & After Rosiglitazone & Difference & $\mathbf{p}^{*}$ & Baseline & \\
\hline P-Homocystine $(\mu \mathrm{mol} / \mathrm{L})$ & $10.3 \pm 2.4$ & $10.6 \pm 2.1$ & $0.3 \pm 1.7$ & 0.612 & $9.5 \pm 1.4$ & 0.408 \\
\hline B-HbAIc (\%) & $5.76 \pm 0.85$ & $5.60 \pm 0.69$ & $-0.24 \pm 0.26$ & 0.035 & $4.46 \pm 0.34$ & $<0.001$ \\
\hline B-Hemoglobin (g/L) & $148 \pm 13$ & $139 \pm 15$ & $-9 \pm 11$ & 0.008 & $145 \pm 14$ & 0.815 \\
\hline P-GGT ( $\mu$ kat/L) & $0.81 \pm 0.57$ & $0.50 \pm 0.26$ & $-0.31 \pm 0.35$ & 0.025 & $0.61 \pm 0.79$ & 0.055 \\
\hline P-ALT ( $\mu$ kat/L) & $0.72 \pm 0.53$ & $0.61 \pm 0.32$ & $-0.10 \pm 0.24$ & 0.260 & $0.59 \pm 0.46$ & 0.606 \\
\hline P-CRP (mg/L) & $4.22 \pm 2.33$ & $3.33 \pm 1.73$ & $-0.89 \pm 2.67$ & 0.295 & $3.67 \pm 0.82$ & 0.864 \\
\hline P-ADMA $(\mu \mathrm{mol} / \mathrm{L})$ & $0.62 \pm 0.06$ & $0.64 \pm 0.06$ & $0.02 \pm 0.04$ & 0.260 & $0.66 \pm 0.10$ & 0.606 \\
\hline P-Glucose (mmol/L) & $8.62 \pm 2.42$ & $6.99 \pm 0.72$ & $-1.63 \pm 2.27$ & 0.008 & $5.18 \pm 0.50$ & $<0.001$ \\
\hline P-Total cholesterol (mmol/L) & $5.11 \pm 1.25$ & $6.26 \pm 1.81$ & $1.15 \pm 0.67$ & 0.008 & $5.05 \pm 0.62$ & 0.423 \\
\hline P-HDL-cholesterol (mmol/L) & $1.07 \pm 0.22$ & $1.19 \pm 0.19$ & $0.12 \pm 0.18$ & 0.110 & $1.52 \pm 0.52$ & 0.093 \\
\hline P-LDL-cholesterol (mmol/L) & $3.11 \pm 1.00$ & $4.24 \pm 1.59$ & $1.13 \pm 0.74$ & 0.008 & $2.84 \pm 0.79$ & 0.351 \\
\hline P-Triglycerides (mmol/L) & $1.86 \pm 0.81$ & $1.81 \pm 0.74$ & $-0.05 \pm 0.37$ & 0.953 & $1.25 \pm 0.37$ & 0.167 \\
\hline S-NEFA $(\mathrm{mmol} / \mathrm{L})$ & $0.27 \pm 0.03$ & $0.18 \pm 0.05$ & $-0.09 \pm 0.05$ & 0.008 & $0.20 \pm 0.07$ & 0.106 \\
\hline P-oxLDL (ng/L) & $250 \pm 171$ & $223 \pm 152$ & $-27 \pm 22$ & 0.008 & $165 \pm 104$ & 0.370 \\
\hline
\end{tabular}

GGT: $\gamma$-glutamyltransferase, ALT: alanine aminotransaminase, CRP: C-Reactive Protein, ADMA: asymmetric dimethylarginine (mean values 8 am - 5 pm), NEFA: non-esterified fatty acids, oxLDL: oxidized-LDL-cholesterol

*Wilcoxon's matched pairs test

** Mann-Whitney U-test

resulted in lower levels of glucose and NEFA, an indirect, but clear sign of increased insulin sensitivity, but this was not paralleled by decreased levels of ADMA.

Despite the relatively small number of subjects investigated in our study, clearly significant results were obtained. A HPLC-method has less coefficient of variation and would have been preferable for the ADMA analysis. Therefore, we can not rule out that a small decrease of ADMA levels after treatment with rosiglitazone, would have been found with a larger study group and/or using a HLPCmethod. Other studies have however shown clearly conflicting results concerning an association between ADMA and insulin resistance and diabetes [15-18]. The picture of ADMA in insulin resistance and diabetes is thus not clear and there is an obvious need for more studies to clarify the complex role of ADMA in diabetes.

As a result of normal protein turnover about $60 \mathrm{mg}$ of ADMA is produced daily. The breakdown of ADMA by the enzymes DDAH1 and DDAH2 appears to be more influenceable than its synthesis [19]. DDAH is among several factors inhibited by high fat-containing meals. In the study of Fard et al, ADMA increased after a high fat meal in 


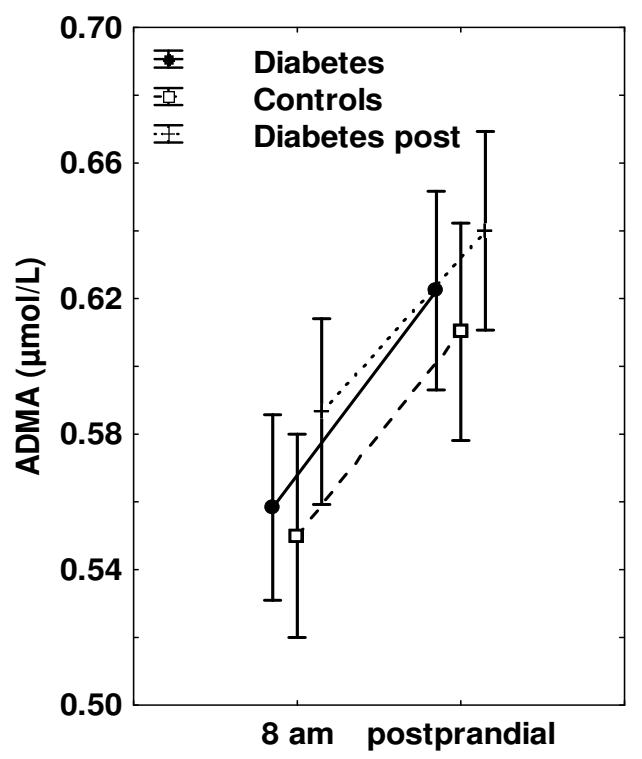

Fig. (1). Daytime diurnal pattern of ADMA (asymmetric dimethylarginine) concentration in patients with diabetes before $(n=6)$ and after intervention $(n=6)$ with rosiglitazone and in controls $(\mathrm{n}=5)$ at 08 am compared to postprandial $(11 \mathrm{am}-5 \mathrm{pm})$. The postprandial values were $9 \%$ higher $(\mathrm{p}=0.006)$ but no significant differences were seen between groups.

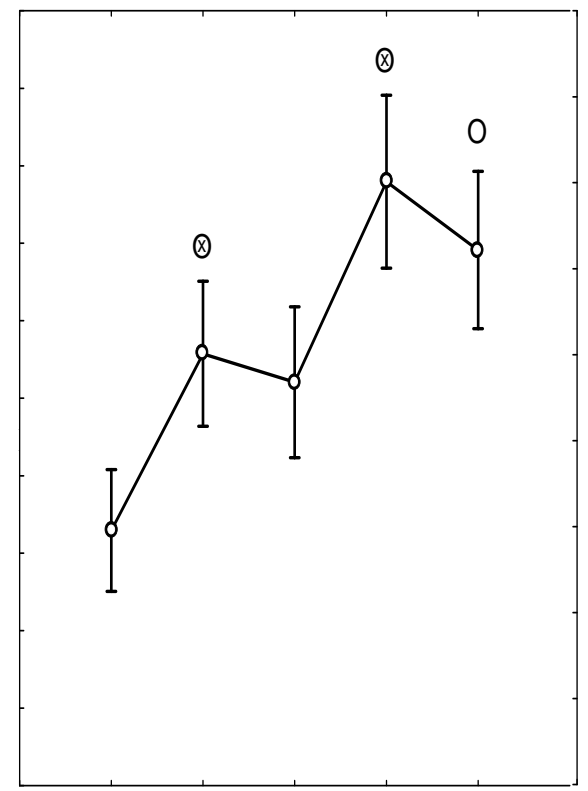

08 am 11 am 1 pm 3 pm 5 pm

Fig. (2). Daytime diurnal pattern of ADMA (asymmetric dimethylarginine) concentration in patients with diabetes before $(n=6)$ and after intervention $(n=6)$ with rosiglitazone and in controls $(\mathrm{n}=5)$, combined $(\mathrm{n}=17), \mathrm{O}=$ statistically significant vs 08 am and $\mathrm{X}=$ statistically significant $v s$ previous value. A statistical significans was seen at $11 \mathrm{am}, 3 \mathrm{pm}$ and $5 \mathrm{pm}$ compared to the mean ADMA value at $08 \mathrm{am}$. A statistical significans was also seen at $3 \mathrm{pm}$ compared to $1 \mathrm{pm}$.

patients with type 2 diabetes [4]. In the present study, with the exception of the morning of the investigation day, the rest of the day was spent in a postprandial state. Thus, the time of day may not be of importance, but whether the subject has eaten once or several times or not at all might be of importance for daytime differences of ADMA concentrations. These differences may in part explain inconsistent results regarding ADMA in studies using plasma samples both obtained in the morning and afternoon. ADMA has been described as very stable with little intraindividual variation [20] and to the best of our knowledge this is a novel finding.

Reference limits calculated for circulating ADMA in our large community-based healthy reference sample confirm the previous observation of a relatively narrow distribution of concentrations. This suggests a tight physiological control of ADMA plasma concentrations, Furthermore, the increase of ADMA after breakfast could correlate with the morning peak in the onset of myocardial infarction [21].

\section{CONCLUSIONS}

In summary, in this short intervention study increasing insulin sensitivity with rosiglitazon in patients with type 2 diabetes, patients showed improved glycemic control with decreased levels of HbA1c, glucose and NEFA but these changes did not correlate to any changed levels of ADMA. No associations were found between NEFA and ADMA levels before or after intervention with rosiglitazon. The study together with other reports [15-18] instead supports a more complex role of ADMA in insulin resistance and diabetes.

The main finding of this study was a clear daytime diurnal pattern of ADMA concentration both in diabetic and non diabetic subjects with average morning concentrations significantly lower than during the rest of the day.

Although the physiological role of ADMA association with food intake and the daytime ADMA variation remains in question, this variation should be taken into account when interpreting ADMA research data.

\section{ACKNOWLEDGEMENTS}

This work was supported by a grant from the Medical Research Council of Southeast Sweden (FORSS). We are also indebted to nurse Agneta Hallengren for excellent assistance.

\section{CONFLICTS OF INTEREST STATEMENT}

The authors declare no conflict of interest.

\section{REFERENCES}

[1] Vallance P, Leone A, Calver A, Collier J, Moncada S. Endogenous dimethylarginine as an inhibitor of nitric oxide synthesis. J Cardiovasc Pharmacol 1992; 20: S60-2.

[2] Harrison DG. Cellular and molecular mechanisms of endothelial cell dysfunction. J Clin Invest 1997; 100: 2153-7.

[3] Sydow K, Mondon CE, Cooke JP. Insulin resistance: potential role of the endogenous nitric oxide synthase inhibitor ADMA. Vasc Med 2005; 10: S35-43.

[4] Fard A, Tuck CH, Donis JA, et al. Acute Elevations of Plasma Asymmetric Dimethylarginine and Impaired Endothelial Function in Response to a High-Fat Meal in Patients With Type 2. Diabetes Arteriosclerosis, Thrombosis, and Vascular Biology 2000; 20: 2039-44.

[5] Surdacki A, Nowicki M, Sandmann J, et al. Reduced urinary excretion of nitric oxide metabolites and increased plasma levels of 
asymmetric dimethylarginine in men with essential hypertension. $\mathbf{J}$ Cardiovasc Pharmacol 1999; 33: 652-8.

[6] Böger RH, Bode-Böger SM, Szuba A. Assymetric dimethylarginine (ADMA): a novel risk factor for endothelial dysfunction: its role in hypercholesterolemia. Circulation 1998; 98: 1842-7.

[7] Vallance P, Leone A, Calver A, Collier J, Moncada S. Accumulation of an endogenous inhibitor of nitric oxide synthesis in chronic renal failure. Lancet 1992; 339: 572-5.

[8] Marliss EB, Chevalier S, Gougeon R, et al. Elevations of plasma methylarginines in obesity and ageing are related to insulin sensitivity and rates of protein turnover. Diabetologia 2006; 49: 351-9.

[9] Koistinen HA, Zierath JR. Regulation of glucose transport in human skeletal muscle. Ann Med 2002; 34: 410-8.

[10] Boden G. Effects of free fatty acids (FFA) on glucose metabolism: significance for insulin resistance and type 2 diabetes. Exp Clin Endocrinol Diabetes 2003; 111: 121-4.

[11] Racette SB, Davis AO, McGill JB, Klein S. Thiazolidinediones enhance insulin-mediated suppression of fatty acid flux in type 2 diabetes mellitus. Metabolism 2002; 51: 169-74.

[12] Stühlinger MC, Abbasi F, Chu JW, Lamenola CMSN, Tracey L. Relationship Between Insulin Resistance and an Endogenous Nitric Oxide Inhibitor. JAMA 2002; 287: 1420-6.

[13] Schofield WN, Schofield C, James WPT. Basal Metabolic Rate: Review and Prediction. Hum Nutr Clin Nutr 1985; 93: 1-96.
[14] Dahlberg G. Statistical methods for medical and biological students. London: George Allen \& Unwin Ltd 1940.

[15] Mittermayer F, Schaller G, Pleiner J, et al. Krzyzanowska K. Rosiglitazone prevents free fatty acid-induced vascular endothelial dysfunction. J Clin Endocrinol Metab 2007; 92: 2574-80.

[16] Kelly AS, Thelen AM, Kaiser DR, Gonzalez-Campoy JM, Bank AJ. Rosiglitazone improves endothelial function and inflammation but not asymmetric dimethylarginine or oxidative stress in patients with type 2 diabetes mellitus. Vasc Med 2007; 12: 311-8.

[17] Teerlink T, Heine RJ, Nijpels G, et al. Asymmetric dimethylarginine (ADMA) is associated with incident cardiovascular disease in the general population. The Hoorn Study. Atherosclerosis Suppl 2006; 7: 23.

[18] Wanby P, Teerlink T, Brudin L, et al. Asymmetric dimethylarginine (ADMA) as a risk marker for stroke and TIA in a Swedish population. Atherosclerosis 2006; 185: 271-7.

[19] Bode-Böger SM, Scalera F, Ignarro LJ. The L-arginine paradox: Importance of the L-arginine/asymmetrical dimethylarginine ratio. Pharmacol Ther 2007; 114: 295-306.

[20] Schwedhelm E, Xanthakis V, Maas R, et al. Asymmetric Dimethylarginine Reference Intervals Determined with Liquid Chromatography-Tandem Mass Spectrometry: Results from the Framingham Offspring Cohort. Clin Chem 2009; 55: 1539- 45.

[21] Muller JE, Stone PH, Turi ZG, et al. Circadian variation in the frequency of onset of acute myocardial infarction. $\mathrm{N}$ Engl J Med $1985 ; 313: 1315-22$

(C) Carlsson et al.; Licensee Bentham Open.

This is an open access article licensed under the terms of the Creative Commons Attribution Non-Commercial License (http://creativecommons.org/licenses/by$\mathrm{nc} / 3.0 /$ ) which permits unrestricted, non-commercial use, distribution and reproduction in any medium, provided the work is properly cited. 